\title{
Surgical Management of Periapical Lesion in Maxilla Using PRF As Graft for Early Wound Healing- A Case Report
}

\author{
Dr. M. Kavitha ${ }^{1}$, Dr.Umesh. G ${ }^{2}$. \\ I' Professor \& Head Of The Department, Dept. Of Conservative Dentistry \& Endodontics, Tamilnadu Govt. \\ Dental College \& Hospital Chennai, India) \\ ${ }^{2}$ ( Post Graduate Student, Dept. Of Conservative Dentistry \& Endodontics, Tamilnadu Govt. Dental College \& \\ Hospital Chennai, India)
}

\begin{abstract}
Periapical surgery is the last resort of the treatment option in the presence of a large periapical granuloma or cyst and the first line idea is to remove periapical pathosis to achieve absolute wound healing and regeneration of soft and hard tissue including periodontium. Platelet rich fibrin (PRF) is a second generation platelet concentration with its greater potency in wound healing and aids in accelerated bone regeneration as it is rich in plenty of growth factors to achieve the structural and functional rehabilitation of the defect. This case report enlightens the use of PRF in early bone regeneration after curetting the lesion in anterior maxilla as a regenerative therapy adjunct to periapical surgery
\end{abstract}

Keywords: Periapical Surgery, Platelet rich fibrin (PRF) Graft, Apicectomy

\section{Introduction}

Unresolved periapical lesions with large bony defect need a special attention apart from conventional surgical treatment alone. It has been a challenge for endodontists to regenerate the periapical bony defects after the periapical surgery. ${ }^{1}$ Cases with lesion size greater than $5 \mathrm{~mm}$ are reported with delayed or altered healing. ${ }^{7}$ In such cases with large periapical lesion, only surgical management with retrograde filling will not help in regeneration of tissues, instead an attempt to give an environment favorable to regeneration will decide the grade of regeneration of the defect. ${ }^{4}$

PRF as a biodegradable scaffold consists of an autologous leukocyte-platelet-rich fibrin matrix, ${ }^{5}$ consists of a tetra molecular structure comprised of cytokines, platelets and stem cells within it ${ }^{2,3}$ favoring microvascularization and epithelial cell migration to the healing site with a sustained release of growth factors in a period between 1 and 4 weeks, providing the environment for wound healing and tissue regeneration. ${ }^{6}$ Plateletrich fibrin increases cell attachment, proliferation and collagen-related protein expression of human osteoblasts. $^{10}$

\section{Case Report}

A 33 year-old male patient reported to the Department of Conservative Dentistry and Endodontics, Tamil Nadu Government Dental College and Hospital with the chief complaint of pain and swelling in upper front tooth region (Fig 1A) and history of root canal treatment in upper anterior tooth. On inspection, tooth 21 was slightly discolored and on palpation, a soft diffuse swelling was present in anterior labial region in relation to 21. Radiographically large periapical radiolucency seen at the apex of 21 with well obturated root canal (Fig 1B). Based on the clinical and radiographic finding, it has been diagnosed as infected periapical granuloma in relaation to 21 .

Periapical surgery was planned with apicectomy followed by retrograde filling. Oral prophylaxis and Routine blood investigations were carried out a day prior to surgery. Patient was prescribed prophylactic antibiotic. Surgical procedure initiated raising a triangular full thickness mucoperiosteal flap using crevicular incisions with limiting vertical incision on distal aspect of 23 under local anesthesia (Fig 2A). Bony defect with loss of labial cortical bone of tooth 21 was seen. Labially the bony window was enlarged, and curettage of the lesion done. Apicectomy was done in 21 and the gutta-percha sealed using heated end of ball burnisher (Fig 2B). Retrograde filling using GIC-GC Fuji IX \& the regenerative scaffold used in this case was PRF (Fig 2C). PRF was prepared by collecting blood without anticoagulant in $10-\mathrm{mL}$ tubes which are immediately centrifuged at $3000 \mathrm{rpm}$ for 10 minutes, placed in the bony defect and labial sutures placed. Postoperative instructions given and antibiotics prescribed. The patient was followed up after 3, 6, \& 12 month (Fig 3A, B \&C). Twelve months post operative radiograph showed satisfactory regeneration of bone in the periapical area of 21 (Fig 3C). 


\section{Discussion}

The Possible explanation of how periapical lesions formed is, as an inflammatory reaction and it may be established due to leakage of irritants from infected root canal into the periradicular tissue. It is very difficult to debride the accessory canals in the apical region of root canal where the number of accessory canals are more which can initiate the periapical lesion even after thorough debridement of the canal and obturating the root canal with excellent coronal and apical seal. Nonsurgical root canal therapy often fails to remove the lesion leaving surgery as the last resort of treatment by which lesion is removed followed by placement of suitable graft material. The present case report evaluated the efficacy of PRF in the treatment of large bony defect and regeneration of lost tissue.

Platelet-rich fibrin as a grafting material eliminating the risk of recipient site morbidity which is major drawback in auto graft and PRF provides consistency that favors clot stability and the membranous shape allows creating a natural "barrier effect" on the bone defect that were opened in the surgical areas. ${ }^{9}$ PRF is a matrix of autologous fibrin with the intrinsic incorporation of cytokines within the fibrin mesh allows for their progressive release upto 7 to 10 days, as the PRF disintigrates. ${ }^{8}$

PRF is a matrix of autologous fibrin rich in GF including platelet derived growth factor (PDGF), transforming growth factor- $\beta$ (TGF- $\beta$, including $\beta$-1and $\beta$-2-isomers), vascular endothelial growth factor (VEGF), and epidermal growth factor ${ }^{10}$. PRF contains all key immune cytokines such as interleukins IL1 $\beta$,IL6,IL-4 and tumour necrosis factor. PRF has a supportive effect on the immune system because it is able to stimulate defense mechanisms. This could be important in the case of wound infection. ${ }^{2}$

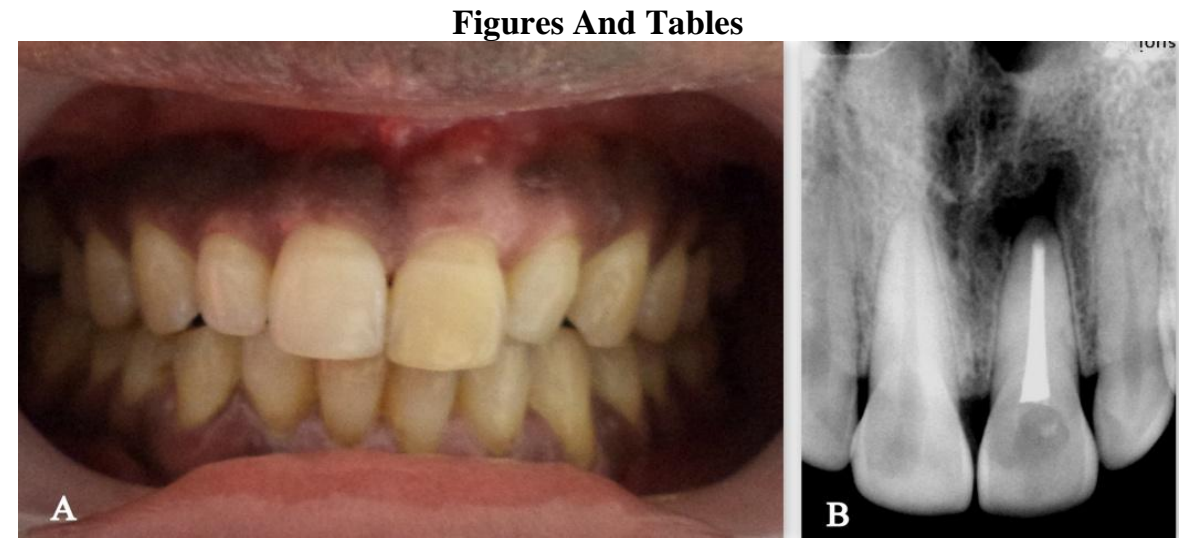

[Fig-1] A. Preoperative Clinical, B. Preoperative Radiograph

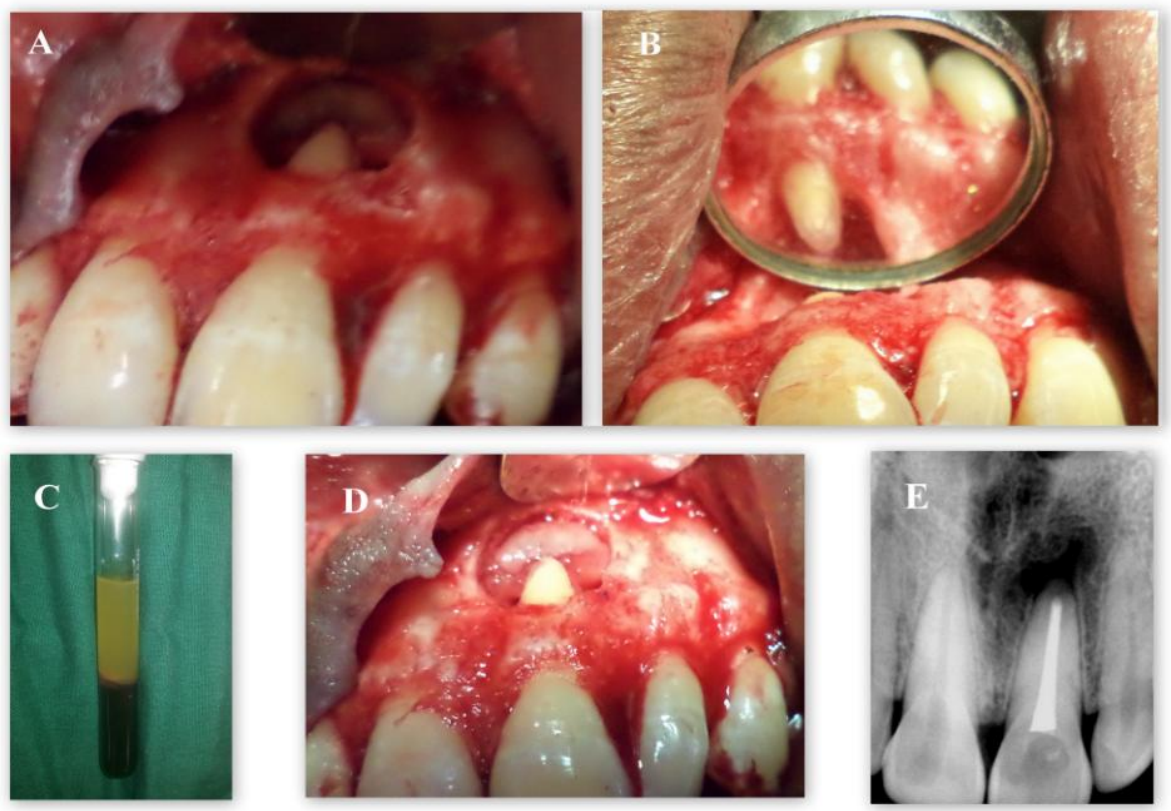

[Fig-2] A. Labial triangular full thickness mucoperiosteal flap raised, B. Apicectomy done in 21, C. PRF, D. Root end filling done with glass ionomer cement in 21 \& PRF Graft placed in the defect, E. Immediate Post operative radiograph 

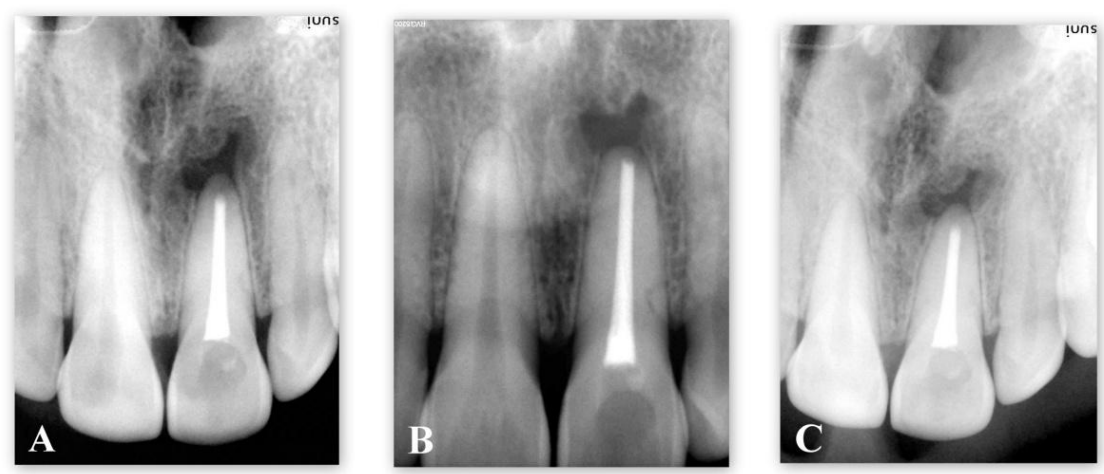

[Fig.3] A. 3 Months Post operative radiograph, B. 6 Months Post operative radiograph, C. 12 Months Post operative radiograph

\section{Conclusion}

The present case report ensures the positive effect of grafting the PRF when used as regenerative therapy adjunct to endodontic surgical procedure. The follow up showed good clinical and radiographic signs of healing and the use of PRF alone as grafting material contributing to successful treatment of such cases. However, further studies are needed to substantiate the use of PRF alone as grafting material.

\section{References}

[1]. Chen FM, Zhang J, Zhang M, An Y, Chen F, Wu ZF. A review on endogenous regenerative technology in periodontal regenerative medicine. Biomaterials 2010;31:7892-927.

[2]. Dohan DM, Choukroun J, Diss A, Dohan SL, Dohan AJ, Mouhyi J and Gogly B. Platelet-rich fibrin (PRF): a second-generation platelet concentrate. Part II: platelet-related biologic features. Oral Surg Oral Med Oral Pathol Oral Radiol Endod 2006; 101: 45-50.

[3]. Gupta V, Bains BK, Singh GP, Mathur A and Bains R. Regenerative potential of platelet rich fibrin in dentistry: Literature review. Asian J Oral Health Allied Sci 2011; 1: 22-28.

[4]. Kim S, Kratchman S. Modern endodontic surgery concepts and practice: A review. J Endod 2006;32(7):601-623.

[5]. Kiran NK, Mukunda KS, Tilak Raj TN. Platelet concentrates: A promising innovation in dentistry. J Dent Sci Res 2011; 2: 50-61.

[6]. Li Q, Pan S, Dangaria SJ, Gopinathan G, Kolokythas A, Chu S, Geng Y, Zhou Y and Luan X. Platelet-rich fibrin promotes periodontal regeneration and enhances alveolar bone augmentation. Biomed Res Int 2013: 638043.

[7]. Rud J, Andreason JO, Jenson JE. A multivariate analysis of various factors upon healing after endodontic surgery. Int J Oral Surg 1972;1(5):258-271.

[8]. Su CY, Kuo YP, Tseng YH, Su CH, Burnouf T. In vitro release of growth factors from platelet-rich fibrin (PRF): A proposal to optimize the clinical applications of PRF. Oral Surg Oral Med Oral Pathol Oral Radiol Endod 2009;108:56-61.

[9]. Tatullo M, Marrelli M, Cassetta M, Platelet Rich Fibrin (P.R.F.) in Reconstructive Surgery of Atrophied Maxillary Bones: Clinical and Histological Evaluations. Int. J. Med. Sci. 2012;9(10):872-880.

[10]. Wu CL, Lee SS, Tsai CH, Lu KH, Zhao JH and Chang YC. Platelet-rich fibrin increases cell attachment, proliferation and collagenrelated protein expression of human osteoblasts. Aust Dent J 2012; 57: 207-212. 